\title{
Poster Abstract: Multi-Channel Communication vs. Adaptive Routing for Reliable Communication in WSNs
}

\author{
Antonio Gonga ${ }^{1}$, Olaf Landsiedel ${ }^{*}$, Pablo Soldati $^{1}$, Mikael Johansson ${ }^{1}$ \\ gonga@kth.se, olafl@chalmers.se, soldati@kth.se, mikaelj@kth.se \\ ${ }^{1}$ KTH Royal Institute of Technology, Sweden \\ ${ }^{2}$ Chalmers University of Technology, Sweden
}

\begin{abstract}
Interference and link dynamics constitute great concerns for stability and performance of protocols in WSNs. In this paper we evaluate the impact of channel hopping and adaptive routing on delay and reliability focusing on delay critical applications.
\end{abstract}

\section{Categories and Subject Descriptors}

C.2.1 [Network Architecture and Design]: Wireless Communication

\section{Keywords}

Wireless Sensor Network, Interference, Channel Hopping

\section{INTRODUCTION}

Interference and link dynamics constitute great concerns for stability and performance of wireless sensor network protocols, especially for delay critical applications [1]. These phenomena normally manifest in link burstiness, i.e, prolonged periods of time where packet transmissions from sender to receiver are lost [10]. Such loss bursts cause delays and instability in communication protocols with potentially severe consequences in, e.g., critical processes in industrial automation [7] and health care $[6,2]$.

A number of papers show that frequency diversity, e.g., by employing channel hopping, increases the resilience to interference and link dynamics $[9,11,12,14]$, while others argue that adaptive routing provides sufficient, or even superior, results [7]. This paper presents our ongoing work on filling this gap. Overall, we believe that it is important to understand the advantages and limitations of channel hopping as it is increasingly adapted by standard bodies such as IEEE 802.15.4e [4], ISA100.11a [5], and WirelessHART [13].

\section{CONTRIBUTION}

Experimental Setup: The data in our analysis is collected from a testbed consisting of 32 TelosB motes scattered throughout the ceiling of offices in the following way: In

\footnotetext{
*Work done while at ACCESS Linnaeus Center, KTH Royal Institute of Technology, Sweden
}

Copyright is held by the author/owner(s). IPSN'12, April 16-20, 2012, Beijing, China. ACM 978-1-4503-1227-1/12/04.

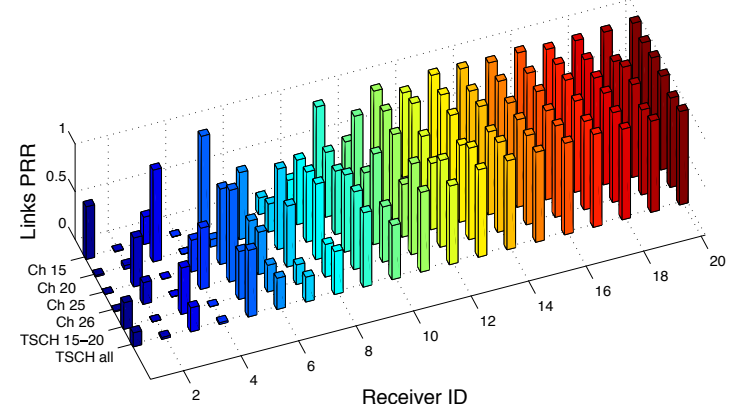

Figure 1: Average links PRR for a random source node to all its neighbors in our testbed: for channels $c_{15}, c_{20}, c_{25}$ and $c_{26}$, and for the hopping sequences $\left\{c_{15}, c_{20}\right\}$ and $\left\{c_{15}, c_{20}, c_{25}, c_{26}\right\}$.

round robin, each node transmits 10,000 consecutive packets with inter-packet interval of $10 \mathrm{~ms}$ (the time slot length used by standards such as WirelessHART). All other nodes log which of these packets they received. We collected traces for three scenarios: single channel, two-channel hopping, and $n$ channel hopping (hopping sequences of increasing length, i.e, $2,4,8,12$, and 16 channels). Figure 1 shows typical results for a single node transmitting 10,000 consecutive packets on ISM-band channels 15, 20, 25 and 26 along with TSCH sequences $\{15,20\}$ and $\{15,20,25,26\}$. The packet reception ratios shown are averaged over 100 consecutive trials.

Experimental Metrics: The intuition behind the use of multi-channel communication is that different channel are subject to different interference patterns. Thus, transmitting a packet on alternating channels decreases the impact of interference and reduces the (re)transmission count until a packet is successfully received. In this paper we evaluate how channel hopping decreases the duration of burst losses and increases their statistical independence, i.e., their $\beta$-factor [10]. $\beta$ quantifies the temporal correlation of packet losses. A value of $\beta=0$ identifies a link with independent packet losses (following a Bernoulli process), while a value of $\beta=1$ indicates a bimodal link, i.e., a link that exists either in a good or a bad state.

Adaptive routing, however, allows the network to adapt its routing topology to interference sources and thus choose next hops that are highly reliable and show only limited impact of interference. We evaluate this claim, and compare 


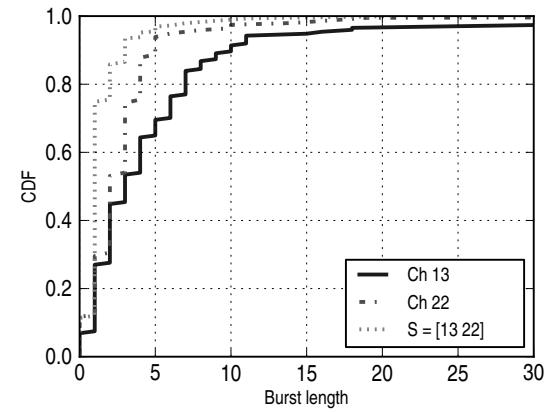

(a) Channel hopping reduces the maxiumum burst loss duration on WIFIinterfered channels.

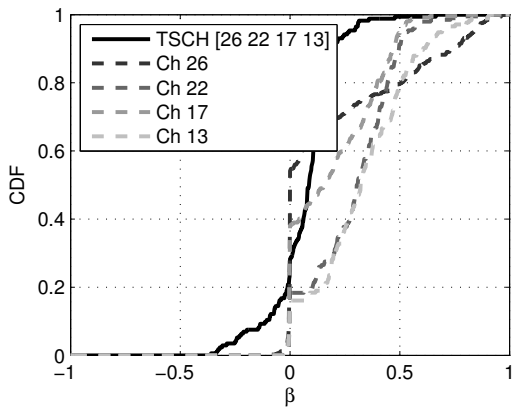

(b) Channel hopping decreases the correlation of packet loss ( $\beta$-factor).

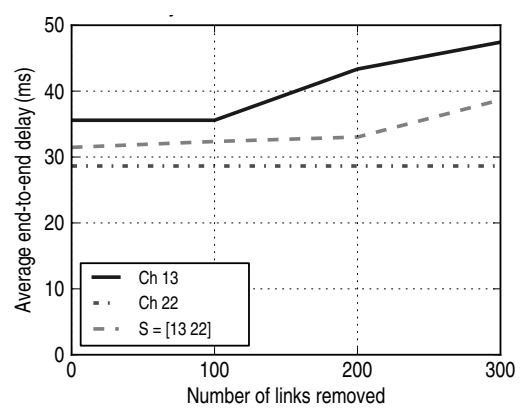

(c) Routing delay: single-channel vs. channel-hopping on Wifi-interfered channels.

Figure 2: Comparing channel hopping and routing in terms of packet loss and delay.

adaptive multi-path routing to channel-hopping in topologies of different densities.

Experimental Results: Figure 2(a) depicts the CDF of the maximum burst loss for two Wi-Fi-interfered channels (we plot links with at least 90\% PRR): It shows that channel hopping reduces the duration of burst losses. Figure 2(b) compares the CDF of $\beta$ for a data set collected with a length- 4 channel hopping sequence $S=\left\{c_{26}, c_{22}, c_{17} c_{13}\right\}$, against the CDF of $\beta$ for data sets collected on each individual channel. The major insight from this result is that channel hopping strongly reduces the packet loss correlation in time, with over $95 \%$ of links having a value of $\beta \leq 0.2$ corresponding to roughly independent packet losses.

In Figure 2(c), we explore the impact of network density on end-to-end reliability and delay. To create networks with varying density, we eliminate links from the experimental traces. Starting from the best link in terms of PRR, we remove links until the network becomes disconnected. We compare the multi-hop end-to-end delay and reliability between the two strongly interfered channels and their channel hopping combination. Routing on top of channel hopping yields an average end-to-end delay that is essentially the average of the delay experience when routing on each individual channel.

\section{DISCUSSION AND CONCLUSIONS}

We analyzed single-channel and multi-channel communication over a single-hop in terms of maximum burst loss, temporal correlation of losses, and end-to-end delay. Our results show that, on a single-hop, multichannel communication via channel hopping significantly reduces link burstiness and decorrelates packet losses in time, to the point that $95 \%$ of links show independent packet losses for hopping sequences with more than two channels. In multi-hop networks, multi-channel communication and adaptive routing yield similar end-to-end reliability in dense and medium dense deployments. This can be explained since routing protocols tend to use good long-term stable links, thus avoiding the intermediate links where burstiness is more dominant.

Overall, our results indicate two key observations: (1) Channel hopping de-correlates packet losses both across time and frequency domain, thus reducing the number of consecutive packet losses compared to the corresponding individual channels. Therefore, in single-hop communication, or on fixed topologies where adaptive routing is not an option (such as preplanned WirelessHART deployments) frequency diversity can yield significant reliability improvements. (2) However, when routing topologies can be adapted to link dynamics and interference, our results indicate that adaptive routing without channel hopping provides on-par reliability. Unless the deployment is very sparse, there are sufficiently many good links on every channel to provide end-to-end connectivity that is comparable with the multi-channel solution, or even better, in terms of end-to-end delay. These paths would readily be found using standard routing protocols, such as CTP [3] or RPL [8].

\section{REFERENCES}

[1] C. A. Boano, T. Voigt, C. Noda, K. Romer, and M. Zuniga. JamLab: Augmenting sensornet testbeds with realistic and controlled interference generation. IPSN'11, April 12-14, 2011, Chicago , IL, 2011.

[2] O. Chipara, C. Lu, T. C. Bailey, and G.-C. Roman. Reliable Clinical Monitoring using Wireless Sensor Networks: Experiences in a Step-down Hospital Unit. ACM Sensys'10, November 3-5, 2010, Zurich, Switzerland, 2010.

[3] O. Gnawali, R. Fonseca, K. Jamienson, D. Moss, and P. Levis. Collection Tree Protocol. ACM Sensys'09, November 4-6, 2009, Berkeley, CA, USA, 2009.

[4] IEEE802154e, Last Access January 2012.

[5] ISA10011a, Last Access January 2012.

[6] J. Ko, C. Lu, M. B. Srivastava, J. A. Stankovic, A. Terzis, and M. Welsh. Wireless Sensor Networks for Healthcare. In Proc. of the IEEE, 2010.

[7] J. Ortiz and D. Culler. Multichannel Reliability Assessment in Real World WSNs. IPSN10, April 12-16, 2010 Stockholm, Sweden, 2010.

[8] RPL Draft 19, Last Access January 2012.

[9] M. Sha, G. Hackmann, and C. Lu. ARCH. Practical Channel Hopping for Reliable Home-Area Sensor Networks.

[10] K. Srinivasan, M. A. Kazandjieva, S. Agarwal, and P. Levis. The $\beta$ Factor: Measuring Wireless Link Burtiness. Sensys 08, 2008.

[11] T. Watteyne, S. Lanzisera, A. Mehta, and K. S. J. Pister. Mitigating Multipath Fading Through Channel Hopping in Wireless Sensor Networks. ICC, 2010, Cape Town, South Africa, 2010.

[12] T. Watteyne, A. Mehta, and K. Pister. Reliability Through Frequency Diversity: Why Channel Hopping Makes Sense. In PE-WASUN, 2009.

[13] WirelessHART, Last Access January 2012.

[14] Y. Wu, J. A. Stankovic, T. He, J. Lu, and S. Lin. Realistic and Efficient Multi-channel Communications in Wireless Sensor Networks. INFOCOM'08, pages 1193 - 1201, 2008. 